\title{
Evidence for field enhanced electron capture by EL2 centers in semi-insulating GaAs and the effect on GaAs radiation detectors
}

\author{
Douglas S. McGregor, ${ }^{a)}$ Ronald A. Rojeski, and Glenn F. Knoll \\ Department of Nuclear Engineering, University of Michigan, Ann Arbor, Michigan 48109 \\ Fred L. Terry, Jr. and Jack East \\ Department of Electrical Engineering and Computer Science, University of Michigan, Ann Arbor, \\ Michigan 48109 \\ Yosef Eisen \\ Soreq Nuclear Research Center, Israel Atomic Energy Commission, Yavne 70600, Israel
}

Received 30 August 1993; accepted for publication 28 February 1994)

\begin{abstract}
The performance of Schottky contact semiconductor radiation detectors fabricated from semi-insulating GaAs is highly sensitive to charged impurities and defects in the material. The observed behavior of semi-insulating GaAs Schottky barrier alpha particle detectors does not match well with models that treat the semi-insulating material as either perfectly intrinsic or as material with deep donors (EL2) of constant capture cross section compensated with shallow acceptors. We propose an explanation for the discrepancy based on enhanced capture of electrons by EL2 centers at high electric fields and the resulting formation of a quasineutral region in the GaAs. Presented is a simple model including field enhanced electron capture which shows good agreement with experimental alpha particle pulse height measurements.
\end{abstract}

\section{INTRODUCTION}

Schottky contact radiation detectors fabricated from bulk GaAs have recently received attention as charged particle and gamma ray spectrometers. ${ }^{1-3}$ Detectors are operated under reverse bias and electron-hole pairs are excited in the active region by absorbed radiation quanta. The subsequent movement of these charge carriers is measured to provide information of the original energy absorbed. ${ }^{4}$ Ideally, Schottky barrier devices should demonstrate a changing active region width (or depletion region) that increases proportionally to $J V .^{5}$ However, previous analysis with detectors fabricated from semi-insulating (SI) liquid encapsulated Czochralski (LEC) bulk GaAs clearly demonstrate that the active region widths do not demonstrate a $J V$ dependence expected from a one-sided Schottky abrupt junction model. ${ }^{3}$ It has been observed that the active region depth demonstrates a nearly linear dependence with applied voltage. ${ }^{6}$

LEC GaAs bulk material is rendered semi-insulating through the use of a careful balance between residual shallow acceptor impurities (mainly carbon) and native defect deep donors EL2. ${ }^{7-9}$ At room temperature and equilibrium, the shallow acceptor impurities are almost completely ionized, however the EL2 native defect deep donors (located approximately $0.8 \mathrm{eV}$ below the conduction band edge) are not fully ionized. The material is rendered semi-insulating provided that

$$
N_{a}>N_{d}
$$

and

$$
N_{\mathrm{DD}}>N_{a}-N_{d}
$$

\footnotetext{
${ }^{\text {a)}}$ Presently located at Sandia National Laboratories, Livermore, CA 94551-
} 0969. where $N_{\mathrm{DD}}$ is the deep donor concentration, $N_{a}$ is the shallow acceptor concentration, and $N_{d}$ is the shallow donor concentration. ${ }^{10}$ An earlier model ${ }^{3,11}$ similar to that of Ryvkin et al. ${ }^{12}$ and Sah and Reddi ${ }^{13}$ was proposed to account for the active region width discrepancy by invoking the ionization of deep donor EL2 centers in Poisson's equation,

$$
-\frac{\partial^{2} V}{\partial x^{2}}=\frac{\partial \mathscr{C}}{\partial x}=\frac{q}{\epsilon_{s}}\left[N_{\mathrm{DD}}^{+}(x)-N_{a}^{-}(x)-n(x)+p(x)\right],
$$

where $N_{a}^{-}$is the shallow acceptor impurity concentration, $N_{\mathrm{DD}}^{+}$is the ionized deep donor concentration, $n$ is the electron concentration, $p$ is the hole concentration, $q$ is the charge of an electron, and $\epsilon_{s}$ is the dielectric constant of the material. The ionized deep donor EL2 concentration is described by

$$
N_{\mathrm{DD}}^{+}=N_{\mathrm{DD}}\left\{1-\left[1+\frac{1}{2} \exp \left(\frac{E_{\mathrm{DD}}-E_{f n}}{k T}\right)\right]^{-1}\right\},
$$

where $N_{\mathrm{DD}}$ is the total deep donor EL2 concentration, $E_{\mathrm{DD}}$ is the deep donor energy level referenced from the conduction band, and $E_{f n}$ is the quasi-Fermi energy referenced from the conduction band.

GaAs material vendors generally report measured shallow acceptor concentrations between $10^{14}$ and $10^{15} / \mathrm{cm}^{3}$ and deep donor EL2 concentrations on the order of $10^{16} / \mathrm{cm}^{3}$. Independent measurements on our GaAs material were performed utilizing an optical transmission technique. ${ }^{14,15}$ The measurements indicated that the material used for our detectors had a total EL2 concentration of approximately $10^{16} / \mathrm{cm}^{3}$ and a neutral EL2 concentration between 8 and $9 \times 10^{15} / \mathrm{cm}^{3} .{ }^{16}$ The difference between the total EL2 concentration and the neutral EL2 concentration gives an estimated shallow acceptor concentration near $10^{15} / \mathrm{cm}^{3}$. Utilizing an average value for the deep donor concentration $\left(N_{\mathrm{DD}}=\mathrm{EL} 2\right.$ density $\approx 10^{16} / \mathrm{cm}^{3}$ ) and the shallow acceptor concentration 
$\left(N_{a} \approx 10^{15} / \mathrm{cm}^{3}\right)$, our previous model indicates that the deep donors would become completely ionized near the reverse biased Schottky junction thus forming a region of positive space charge defined by the net positive charge concentration $\left(N_{\mathrm{DD}}-N_{a}\right) .{ }^{3.6}$ The high concentration of ionized EL2 centers would cause the electric field to drop dramatically near the Schottky contact. The same model was later adopted by a different group with similar results. ${ }^{17}$ Our previous model indicates that several thousand volts are required to fully extend the active region across a $100-\mu$ m-thick device, ${ }^{6}$ however alpha particle irradiation experiments have shown that the active region extends across a $100 \mu \mathrm{m}$ device with only $100 \mathrm{~V}^{3.6}$ Although the model demonstrated that ionized deep donors could severely affect the electric field distribution in such a device, it failed to adequately describe the width of the active region actually observed in experiments.

The purpose of this paper is to propose an explanation for the discrepancy between experimental alpha particle irradiation data and the calculated responses utilizing electric fields based on Eqs. (3) and (4). The explanation is based on two observations. The first is that the native defect EL2 has been shown to undergo a dramatic increase in its electron capture cross section at electric fields near $10^{4} \mathrm{~V} / \mathrm{cm} .{ }^{18,19}$ The second is that the electron emission rate requires higher electric fields $\left(2 \times 10^{5} \mathrm{~V} / \mathrm{cm}\right)$ before it dramatically increases. ${ }^{20}$ There is an indication that injected charge through a forward biased ohmic contact can supply enough carriers to fill the EL2 deep donor centers and produce a neutralization effect. ${ }^{21}$ At electric fields approaching $10^{4} \mathrm{~V} / \mathrm{cm}$, the electron capture rate may be substantially higher than the electron emission rate and the leakage current through a reverse biased Schottky contact may allow for the formation of a quasineutral region similar to that described for Au-doped $\mathrm{Si}^{22}$

\section{FIELD ENHANCED CAPTURE CROSS SECTION ACTIVE REGION MODEL}

The previous model described by Eqs. (3) and (4) assumes that the deep donor levels above the $n$ quasi-Fermi level will be empty (positive ions), while those falling below the $n$ quasi-Fermi level will be filled (neutral). However, the relation is generally used to describe near equilibrium conditions and does not take into account the possible change in emission and capture rates of carriers by the trapping center. It is clear that the detector active region is not at equilibrium at high applied voltages. The steady state ionized deep donor concentration can be approximated by ${ }^{23}$

$$
N_{\mathrm{DD}} \approx \frac{N_{\mathrm{DD}}^{+}}{1+n \sigma_{n} v_{e} / e_{n}},
$$

where $v_{e}$ is the electron velocity, $\sigma_{n}$ is the deep donor electron capture cross section (in $\mathrm{cm}^{2}$ ), and $e_{n}$ is the deep donor electron emission rate per second. If the thermionic leakage current through the reverse biased Schottky contact is substantial, then the deep donors will no longer be fully ionized. This case may account for the observed reduction in the measured EL2 concentration with increased leakage current. ${ }^{24}$

The room temperature capture cross section has been reported to increase from approximately $10^{-15}$ to approxi- mately $10^{-13} \mathrm{~cm}^{2}$ at electric fields near $10^{4} \mathrm{~V} / \mathrm{cm} .{ }^{18.19}$ The room temperature electron emission rate for EL2 is approximately 0.1 per second at electric fields below $10^{5}$ $\mathrm{V} / \mathrm{cm} \cdot{ }^{20,23,24}$ An average leakage current density (measured from our devices) of $8 \mathrm{nA} / \mathrm{mm}^{2}$ results in an electron density $(n)$ for a saturation velocity of $10^{7} \mathrm{~cm} / \mathrm{s}$ approximately equal to $5 \times 10^{5} / \mathrm{cm}^{3}$ flowing through the detector active region. Equation (5) gives $N_{\mathrm{DD}}^{+} \approx 0.95 N_{\mathrm{DD}}$ at low electric fields when the capture cross section is near $10^{-15} \mathrm{~cm}^{2}$, thus demonstrating that most of the EL2 deep donors should be ionized. However, at electric fields approaching $10^{4} \mathrm{~V} / \mathrm{cm}$ where the electron capture cross section increases near that of $10^{-13}$ $\mathrm{cm}^{2}$, the ionized EL2 concentration decreases to $0.17 N_{\mathrm{DD}}$. Therefore, the ionized EL2 concentration decreases to the same order of magnitude as the ionized shallow acceptor concentration. This condition supports the plausibility of a quasineutral region as a result of electric field enhanced capture of electrons. It is hypothesized that as the reverse leakage current (or $n$ ) and $\sigma_{n}$ increase with electric field, a condition is eventually reached in which the difference between the ionized donor and acceptor concentrations becomes very small, hence producing a region of low net space charge or a quasineutral region. We propose that such an effect may in fact help explain the observed results from charged particle analysis in previous work. ${ }^{3}$ If the EL2 deep levels are strongly capturing electrons either injected through the Schottky contact or generated from the valence band, then the assumption of full ionization of the EL2 centers becomes questionable at high electric fields. Unfortunately, to the knowledgc of the authors, no data are readily available describing the EL2 electron capture cross section at electric fields between 0 and $10^{4} \mathrm{~V} / \mathrm{cm}$, hence no empirical formulation of the capture cross section dependence on electric fields is derivable. A model has been proposed incorporating intervalley scattering (from the $\Gamma$ valley to the $L$ valley) as an indication of the expected capture cross section dependence, ${ }^{25}$ however assumptions were made in the model to account for the lack of data. The model described in the literature does indicate that the expected capture cross section should increase dramatically as the electric field approaches some critical value. ${ }^{25}$

\section{A. Electric field model with field enhanced electron capture}

A dramatic increase in electron capture cross section would create a similarly dramatic decrease in ionized EL2 centers. The enhanced capture effect is modeled here by altering Eq. (4) so that the ionization of the deep levels decreases dramatically at electric fields approaching a critical electric field $\left(\mathscr{E}_{c}\right)$. The density of ionized EL2 centers is modeled as

$$
\begin{aligned}
N_{\mathrm{DD}}^{+}= & N_{\mathrm{DD}}\left\{1-\left[1+\frac{1}{2} \exp \left(\frac{E_{\mathrm{DD}}-E_{\mathrm{Fn}}}{k T}\right)\right]^{-1}\right\} \\
& -\int\left(N_{\mathrm{DD}}, \varnothing\right),
\end{aligned}
$$

where $f\left(N_{\mathrm{DD}}, C\right)$ is a filling function that strongly increases the number of filled EL2 traps as the electric field surpasses 


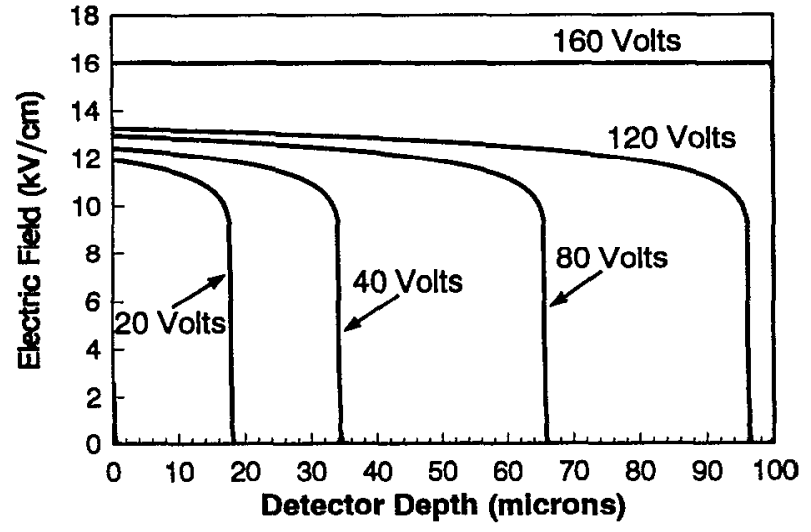

FIG. 1. Numerically calculated electric fields for a 100 - $\mu$ m-thick bulk GaAs detector at different reverse voltages with $\zeta_{c}=7.0 \times 10^{3} \mathrm{~V} / \mathrm{cm}$. The value of $\alpha$ for the calculation was 15 in order to simulate rapid deep donor filling as the electric field approached $10^{4} \mathrm{~V} / \mathrm{cm}$. The filling of deep donors allows for the formation of a fairly constant electric field region. The detector depth refers to the distance from the reverse biased Schottky contact. The electric field magnitude is represented in absolute value.

$\varnothing_{c}$. Since the actual filling function is unknown, an arbitrary function was chosen to represent the rapid filling of the deep donor centers at fields above $\mathscr{Z}_{c}$,

$$
\begin{aligned}
N_{\mathrm{DD}}^{+}= & N_{\mathrm{DD}}\left\{1-\left[1+\frac{1}{2} \exp \left(\frac{E_{\mathrm{DD}}-E_{F n}}{k T}\right)\right]^{-1}\right. \\
& -\left(N_{\mathrm{DD}}-N_{a}\right)\left(\frac{1}{1+\left(\mathscr{E}_{c} / \mathscr{E}\right)^{\alpha}}\right)
\end{aligned}
$$

where the term $\left(N_{\mathrm{DD}}-N_{a}\right)$ ensures that the net space charge in the active region cannot change from a net positive charge to a net negative charge. The value of $\alpha$ determines how rapidly the filling occurs with the changing electric field.

The electric field for a $100-\mu \mathrm{m}$-thick device was calculated by inserting Eq. (7) into Eq. (3). As shown in Fig. 1, the calculated electric field profile demonstrates the appearance of two distinct regions. In the first region, the electric field remains practically constant up to an extended distance into the detector bulk. In the second region, the electric field drops abruptly due to the high concentration of ionized deep donors in a lower field region. Although Fig. 1 shows only the result for $\delta_{c}=7.0 \times 10^{3} \mathrm{~V} / \mathrm{cm}$ and $\alpha=15$, the characteristic shape occurred regardless of the values chosen for $Z_{c}$ and $\alpha$.

\section{B. Schottky barrier solid state detector operation}

If we neglect trapping and recombination, the interaction of alpha particles in a solid state detector can be described briefly as follows. Alpha particles entering a detector excite electron-hole pairs according to the Bragg distribution. ${ }^{26}$ The charge liberated is linear with respect to energy lost by the alpha particle. The charge in the active region of the detector is measured, hence giving a relative measure of the energy deposited in the active region. At zero bias voltage the active region is formed only by the Schottky contact potential resulting in a very thin active region. Referring to Fig. 2(a), the

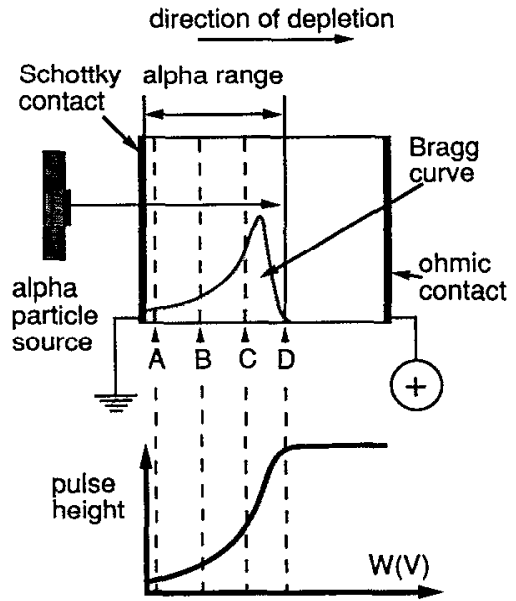

(a)

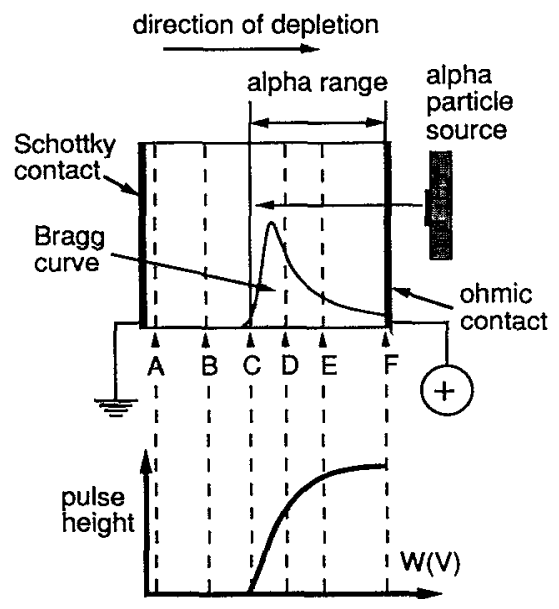

(b)

FIG. 2. Expected voltage dependent pulse height responses to alpha particles from an extrinsic Schottky barrier diode detector. Depicted are the (a) expected pulse height response due to front side irradiation (Schottky contact) and (b) the expected pulse height response due to backside irradiation (ohmic contact). Notice that pulses will be observed at low voltages for front side irradiation, however pulses will not be observed from backside irradiation until a threshold voltage is reached.

charge measured by alpha particles entering the detector front (Schottky contact) would be that produced in the region between the Schottky contact and point $\mathrm{A}$, hence resulting in a low pulse height. Application of voltage extends the active region from point $A$ to point $B$, and the integrated charge in the active region increases, thus producing larger pulse heights. Higher bias voltages increase the active region width resulting in even larger pulse heights until the active region extends beyond point $D$. As the active region extends beyond point $\mathrm{D}$, the total charge liberated by the alpha particle is produced entirely within the active region and the measured charge should reflect the full energy of the original alpha particle.

When the same detector at zero bias voltage is irradiated from the back [Fig. 2(b)], the active region does not extend into the alpha particle range and no pulses are observed. 


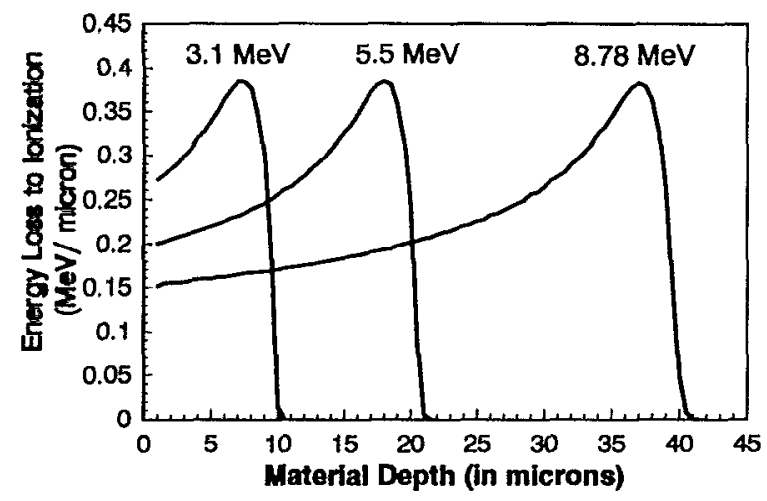

FIG. 3. Bragg ionization curves for $3.1,5.5$, and $8.78 \mathrm{MeV}$ alpha particles in GaAs.

Pulses will not be observed until sufficient voltage is applied to extend the active region to point $\mathrm{C}$. Since a large fraction of the alpha particle energy is deposited near the end of its path, the pulse height will increase abruptly as the active region crosses point $C$. The measured pulse heights will reach their maximum value when the detector becomes fully depleted (point F). Carrier recombination during the detector plasma time $\mathrm{e}^{27.28}$ and carrier trapping will cause the pulse height curves to elongate and saturate at lower pulse height values, however their characteristic shapes will be preserved.

\section{Alpha particle response model}

The response to front and back contact alpha particle irradiation was modeled as follows. The Bragg ionization distribution was used to estimate the alpha particle energy deposition per unit path length. ${ }^{29}$ The ranges of $3.1,5.5$, and $8.78 \mathrm{MeV}$ alpha particles in GaAs are approximately 10,20 , and $40 \mu \mathrm{m}$, respectively (Fig. 3). For the calculation, the electron and hole mean free transit times (due to both trapping and recombination during transit) were assumed to be $10 \mathrm{~ns}^{30}$ Carrier trapping and recombination during transit were taken into account for the calculated pulse height, although pulse height lowering due to recombination during the plasma time was not considered. Vendor supplied measurements of the Hall mobilities were $7500 \mathrm{~cm}^{2} / \mathrm{V} \mathrm{s}$ for electrons and $350 \mathrm{~cm}^{2} / \mathrm{V}$ for holes. The electron and hole velocities as a function of electric field and mobility were taken into account for the calculation. The values of $\xi_{c}$ and $\alpha$ were maintained at $7.0 \times 10^{3} \mathrm{~V} / \mathrm{cm}$ and 15 , which simulates strong deep donor filling as the clectric field approaches $10^{4} \mathrm{~V} / \mathrm{cm}$. The device simulated is a reverse biased Schottky contact diode with the opposite contact being an $n$-type ohmic contact. Positive voltage is applied to the ohmic contact with respect to the Schottky contact. The GaAs material simulated is semi-insulating with an EL2 concentration of $10^{16} / \mathrm{cm}^{3}$ located at $0.8 \mathrm{eV}$ below the conduction band and a shallow acceptor concentration of $10^{15} / \mathrm{cm}^{3}$ assumed to be completely ionized. The equilibrium Fermi level was pinned near midgap in the material bulk and was pinned at $0.8 \mathrm{eV}$ below the conduction band at the Schottky contact interface.

Utilizing electric fields calculated from Eqs. (3) and (7), the responses to $3.1,5.5$, and $8.78 \mathrm{MeV}$ alpha particles were
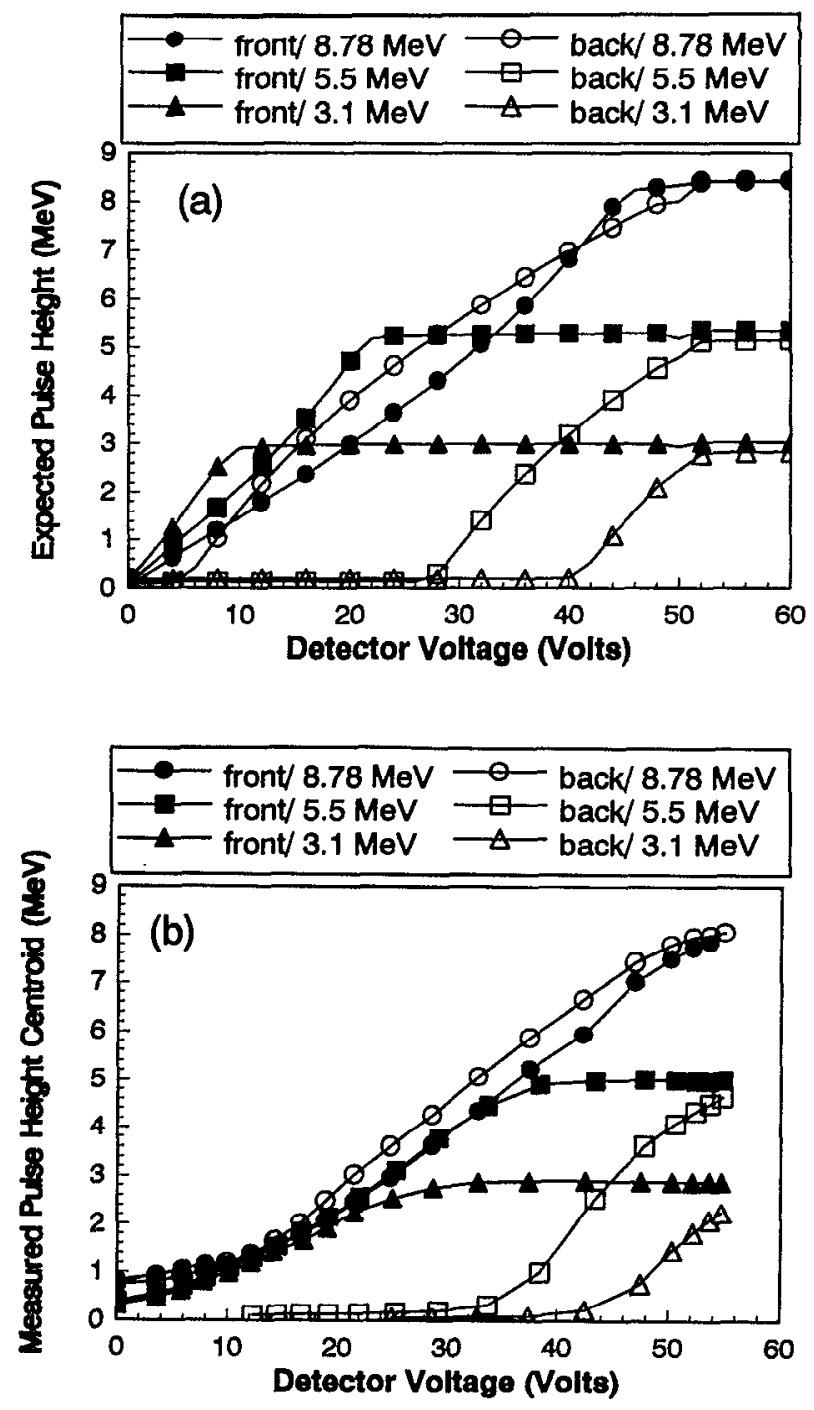

FIG. 4. Comparison of front and back irradiation with alpha particles for a $45-\mu \mathrm{m}$-thick bulk GaAs detector. Shown are (a) the numerically calculated pulse height responses and (b) the measured pulse height responses. The alpha particle energies are $3.1,5.5$, and $8.78 \mathrm{MeV}$. $\mho_{c}$ is $7.0 \times 10^{3} \mathrm{~V} / \mathrm{cm}$ and $\alpha$ is 15 . The pulse height response is shown in terms of energy (MeV) measured from the detector.

calculated for a $45 \mu \mathrm{m}$ detector and a $100 \mu \mathrm{m}$ detector as shown in Figs. 4(a) and 5(a), respectively. From the calculation, if the detector is irradiated from the Schottky contact (front) the pulse height immediately increases with the application of voltage. The voltage at which the pulse height saturates is a function of the particle energy (or range). Ohmic contact (back) irradiation indicates that a threshold voltage must be reached before pulses are observed for particles whose ranges do not extend fully across the device.

\section{Experimental measurements}

GaAs Schottky barrier detectors fabricated in our laboratory were irradiated in vacuum with alpha particle sources with energies of $3.18,5.5$, and $8.78 \mathrm{MeV}$. The detectors under test were Schottky-ohmic devices with positive bias applied to the ohmic contact with respect to the Schottky contact. Energy calibration of the multichannel analyzer was 

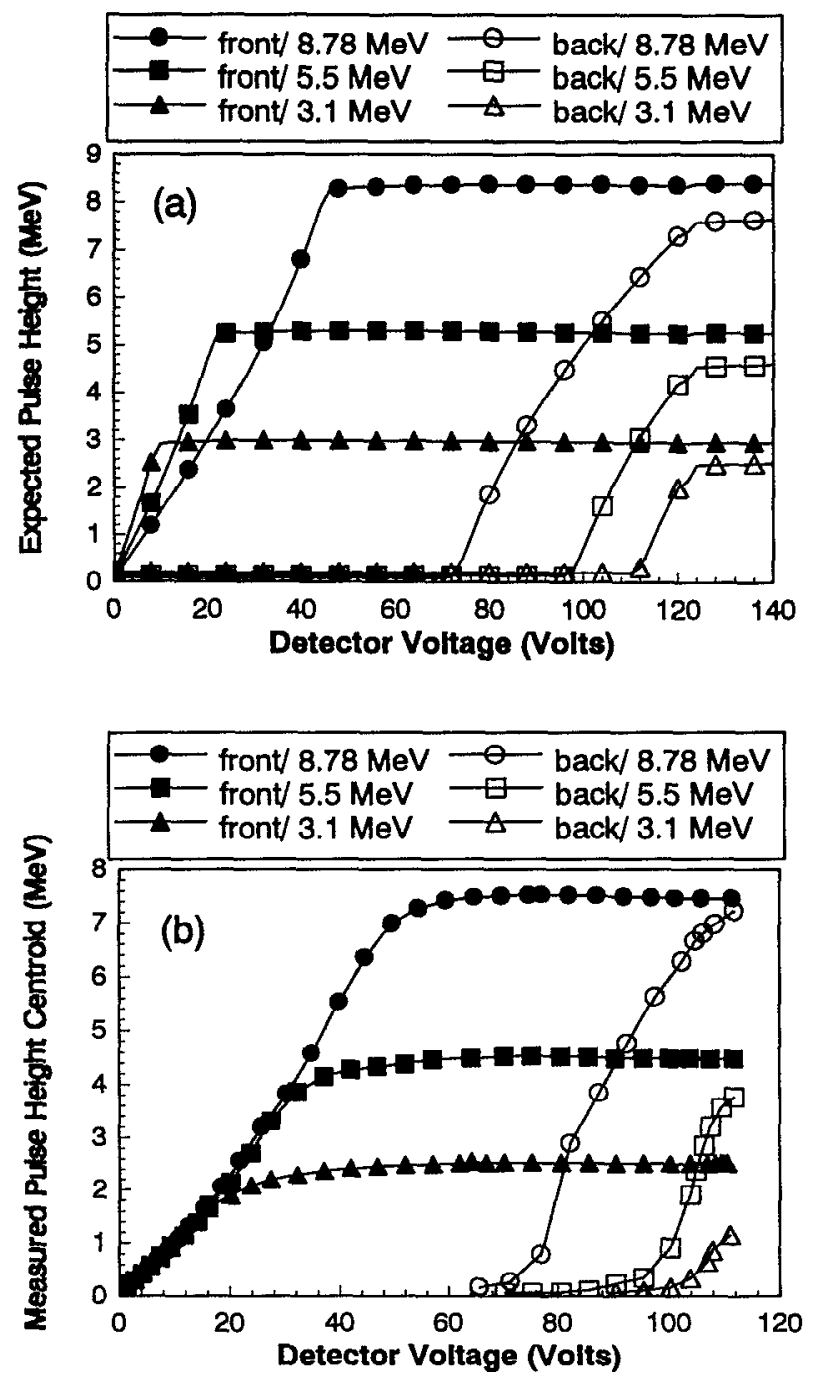

FIG. 5. Comparison of front and back irradiation with alpha particles for a $100-\mu \mathrm{m}$-thick bulk GaAs detector. Shown are (a) the numerically calculated pulse height responses and (b) the measured pulse height responses. The alpha particle energies are $3.1,5.5$, and $8.78 \mathrm{Mev} . \zeta_{c}$ is $7.0 \times 10^{3} \mathrm{~V} / \mathrm{cm}$ and $\alpha$ is 15 . The pulse height response is shown in terms of energy (MeV) measured from the detector.

performed with a silicon surface barrier detector and three spectroscopic grade alpha particle sources, those being ${ }^{148} \mathrm{Gd}(3.18 \mathrm{MeV}),{ }^{241} \mathrm{Am}(5.5 \mathrm{MeV})$, and ${ }^{228} \mathrm{Th}(5.3-8.78$ $\mathrm{MeV}$ ). Figure 6 shows an alpha particle pulse height spectrum from a ${ }^{228} \mathrm{Th}$ source for Schottky contact irradiation of a fully depleted $100 \mu \mathrm{m}$ GaAs detector.

It is difficult to know the actual active region width at any given bias voltage. Therefore, the channel numbers of the alpha particle pulse height spectrum centroids were recorded as a function of voltage. The detector voltage was corrected for the voltage dropped across the detector high voltage bias resistor due to leakage current. The average absorbed energy represented by the pulse height centroid was corrected from that of Si (3.6 eV/e-h pair) to that of GaAs $\left(4.2 \mathrm{eV} / \mathrm{e}-\mathrm{h}\right.$ pair) ${ }^{30}$ For instance, the centroid channel number of the $8.78 \mathrm{MeV}$ alpha particle peak shown in Fig. 6 was recorded as a function of voltage appearing across the detector terminals. The channel numbers were then converted to

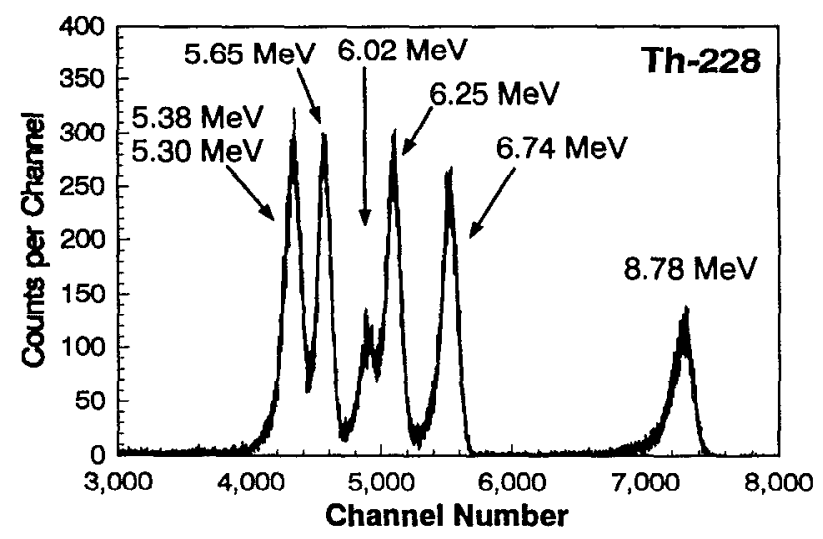

FIG. 6. Alpha particle differential pulse height spectrum from ${ }^{228} \mathrm{Th}$ taken with a $100 \mu \mathrm{m}$ bulk GaAs detector. The detector was irradiated in vacuum from the front contact (Schottky contact).

energy utilizing the Si detector calibration and corrected with the $\mathrm{GaAs} / \mathrm{Si}$ ionization energy ratio. As can be seen, the pulse height responses for 45 and $100 \mu \mathrm{m}$ detectors indicated by the model [Figs. 4(a) and 5(a)] are very similar to experimentally observed results shown in Figs. 4(b) and 5(b). A direct comparison of the modeled results and the measured results for a $100 \mu \mathrm{m}$ detector is shown in Fig. 7.

The voltage at which the alpha particle pulse height begins to increase under backside irradiation gives a measure of the active region depth [point $\mathrm{C}$ in Fig 2(b)]. The active region width is approximated by subtracting the alpha particle range from the total detector width. The experimental results from $45-$ and $100-\mu \mathrm{m}$-thick detectors indicate a nearly linear relationship between detector voltage and active region depth. The active region depth can also be approximated under front side irradiation by observing the voltage at

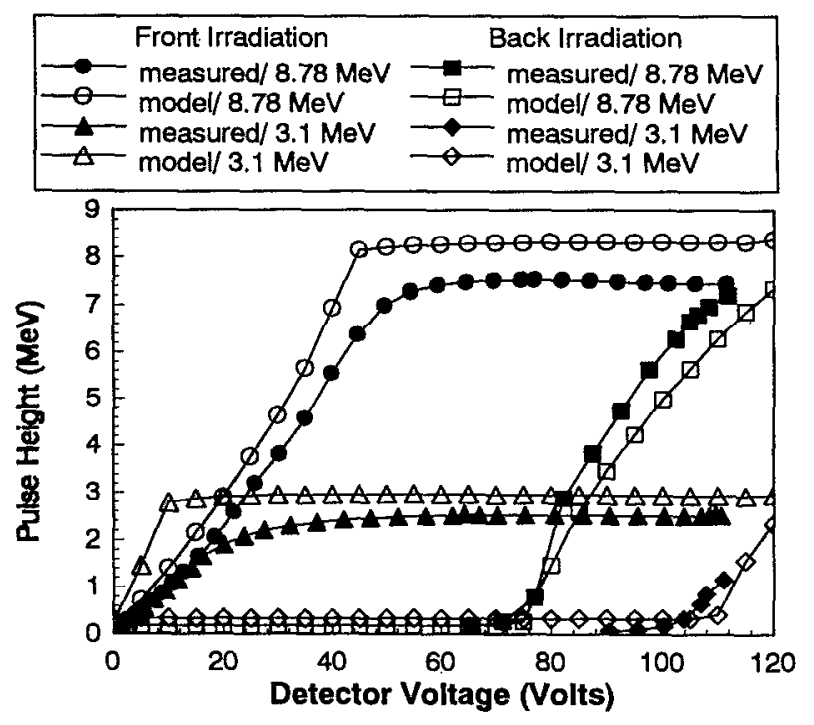

FIG. 7. Overlay comparison between modeled and measured pulse height curves for a $100-\mu \mathrm{m}$-thick bulk GaAs detector. Shown are the curves for 3.1 and $8.78 \mathrm{MeV}$ alpha particles where the pulse height response is shown in terms of MeV. $\varnothing_{c}$ is $7.0 \times 10^{3} \mathrm{~V} / \mathrm{cm}$ and $\alpha$ is 15 for the modeled pulse height curves. 
which the pulse height reaches its near maximum value, however it is expected that the approximation is subject to error due to plasma time charge carrier recombination effects. The observed active region depths are too wide to be accounted for by the dopant concentration involved, and therefore a substantial neutral region (or low space charge region) must be present. Other models invoking deep donor ionization with applied bias without including field enhanced capture effects do not match well with experimental measurements. 6.31

\section{CONCLUDING REMARKS}

We propose that the field enhancement of the EL2 capture cross section coupled to a low emission rate promotes the formation of a quasineutral region at high bias voltages. At low fields, the electron capture rate is low due to a small electron capture cross section value and the ionized EL2 concentration is approximated well by Eqs. (3) and (4). However, at higher applied bias voltages that result in electric fields near $10^{4} \mathrm{~V} / \mathrm{cm}$, the EL2 electron capture rate increases such that the capture rate is substantially higher than the emission rate. ${ }^{18,19,20,24}$ Hence, the electron emission rate becomes the rate limiting process rather than the electron capture rate. The proposed result is the production of a quasineutral region similar to that described for Au-doped $\mathrm{Si}^{22}$ Direct measurements of the potential across similar $\mathrm{GaAs}$ detectors indicated that a flat electric field region was present followed by a sharp drop in electric field strength, ${ }^{32}$ which also indicates the formation of a quasineutral region and helps to support our hypothesis. It is not assumed that the EL2 centers remain filled, but that the time averaged effect of the EL2 centers filling and emptying produces a quasineutral region at sufficiently high fields. Although an arbitrary filling function was selected to represent the rapid filling of EL2 centers at high electric fields, the model qualifies the argument that field enhanced electron capture by the EL2 deep donors can produce a quasineutral region. The model does not take into account changes in the reverse bias leakage current with applied voltage or plasma time recombination effects, which could account for the discrepancies between the modeled results and measured results. However, by invoking the production of a quasineutral region, our model closely reproduces the measured alpha particle pulse height curves shown in Figs. 4(b) and 5(b).

\section{ACKNOWLEDGMENTS}

We thank Dr. Millard G. Mier of Wright Patterson Air Force Base for providing measurements of the neutral and total EL2 densities of the bulk GaAs material investigated.
We also express gratitude towards Dr. David C. Look at Wright State University for helpful discussions concerning the electrical properties of SI GaAs. This project has been funded in part by Los Alamos National Laboratory Subcontract No. 9-XGO-K-7910-1.

${ }^{1}$ T. J. Sumner, S. M. Grant, A. Bewick, J. P. Li, K. Smith, and S. P. Beaumont, Nucl. Instrum. Methods A 322, 514 (1992).

${ }^{2}$ K. W. Benz, R. Irsigler, J. Ludwig, J. Rosenzweig, K. Runge, F. Schäfer, J. Schncider, and M. Webel, Nucl. Instrum. Methods A 322, 493 (1992).

${ }^{3}$ D. S. McGregor, G. F. Knoll, Y. Eisen, and R. Brake, IEEE Trans. Nucl. Sci. NS-39, 1226 (1992).

${ }^{4}$ G. F. Knoll and D. S. McGregor, Mater. Res. Soc. Symp. Proc. 302, 3 (1993).

${ }^{5}$ S. M. Sze, Physics of Semiconductor Devices, 2nd ed. (Wiley, New York, 1981).

${ }^{6}$ D. S. McGregor, Ph.D. dissertation, University of Michigan, 1993.

${ }^{7}$ G. M. Martin, J. P. Farges, G. Jacob, J. P. Hallais, and G. Poiblaud, J. Appl. Phys. 51, 2840 (1980).

${ }^{8} \mathrm{R}$. K. Willardson and A. C. Beer, Semiconductors and Semimetals (Academic, Orlando, FL, 1984), Vol. 20.

${ }^{9}$ J. C. Bourgoin, H. J. von Bardeleben, and D. Stievenard, J. Appl. Phys. 64, R65 (1988).

${ }^{10}$ J. V. DiLorenzo and D. D. Khandelwal, GaAs FET Principles and Technology (Artech House, Dedham, 1982).

${ }^{11}$ D. S. McGregor, G. F. Knoll, Y. Eisen, and R. Brake, Nucl. Instrum. Methods A 322, 487 (1992).

${ }^{12}$ S. M. Ryvkin, L. L. Makovsky, N. B. Strokan, V. P. Subashieva, and A. Kh. Khusainov, IEEE Trans. Nucl. Sci. NS-15, 226 (1968).

${ }^{13}$ C. T. Sah and V. G. K. Reddi, IEEE Trans. Electron Devices ED-11, 345 (1964).

${ }^{14}$ S. K. Brierley and D. S. Lehr, Appl. Phys. Lett. 55, 2426 (1989).

${ }^{15}$ M. G. Mier, D. C. Look, D. C. Walters, and D. L. Beasley, Solid-State Electron. 35, 319 (1992).

${ }^{16}$ M.G. Mier (private communication, Wright Patterson Air Force Base, 1992).

${ }^{17}$ S. P. Beaumont et al., Nucl. Instrum. Methods A 326, 313 (1993).

${ }^{18}$ V. Ya. Prinz and B. A. Bobylev, Sov. Phys. Semicond. 14, 1097 (1980).

${ }^{19}$ V. Ya. Prinz and S. N. Rechkunov, Phys. Status Solidi B 118, 159 (1983).

${ }^{20}$ S. Makram-Ebeid, Mater. Res. Soc. Symp. Proc. 2, 495 (1981).

${ }^{21}$ J. C. Adams, C. D. Capps, R. A. Falk, and S. G. Ferrier, Appl. Phys. Lett. 63, 633 (1993).

${ }^{22}$ P. T. Panousis, R. H. Krambeck, and W. C. Johnson, Appl. Phys. Lett. 15, 79 (1969).

${ }^{23}$ D. C. Look, Electrical Characterization of GaAs Materials and Devices (Wiley, New York, 1989).

${ }^{24}$ S. T. Pantelides, Deep Centers in Semiconductors, 2nd ed. (Gordon and Breach, Philadelphia, 1992).

${ }^{25}$ D. A. Johnson, Ph.D. dissertation, Arizona State University (1989).

${ }^{26}$ R. D. Evans, The Atomic Nucleus (Krieger, Malabar, 1955).

${ }^{27}$ G. Dearnaley and D. C. Northrop, Semiconductor Counters for Nuclear Radiations (Spon, London, 1964).

${ }^{28}$ A. A. Ouaranta, A. Taroni, and G. Zanarini, IEEE Trans. Nucl. Sci. NS-15, 373 (1968).

${ }^{29}$ J. F. Ziegler and J. P. Biersack, TRIM-90, Version 90.05, 1990.

${ }^{30}$ E. Sakai, Nucl. Instrum. Methods 196, 121 (1982).

${ }^{31}$ D. S. McGregor, R. A. Rojeski, G. F. Knoll, F. L. Terry, Jr., J. East, and Y. Eisen, Nucl. Instrum. Methods (in press).

${ }^{32}$ K. Berwick, M. K. Brozel, C. M. Buttar, M. Cowperthwaite, and Y. Hou, Mater. Res. Soc. Symp. Proc. 302, 363 (1993). 